\title{
Localization Accuracy of Track-before-Detect Search Strategies for Distributed Sensor Networks
}

\author{
Thomas A. Wettergren and Michael J. Walsh \\ Naval Undersea Warfare Center, 1176 Howell Street, Newport, RI 02841, USA \\ Correspondence should be addressed to Thomas A. Wettergren, t.a.wettergren@ieee.org
}

Received 22 March 2007; Revised 29 June 2007; Accepted 30 August 2007

Recommended by Frank Ehlers

The localization accuracy of a track-before-detect search for a target moving across a distributed sensor field is examined in this paper. The localization accuracy of the search is defined in terms of the area of intersection of the spatial-temporal sensor coverage regions, as seen from the perspective of the target. The expected value and variance of this area are derived for sensors distributed randomly according to an arbitrary distribution function. These expressions provide an important design objective for use in the planning of distributed sensor fields. Several examples are provided that experimentally validate the analytical results.

Copyright (c) 2008 T. A. Wettergren and M. J. Walsh. This is an open access article distributed under the Creative Commons Attribution License, which permits unrestricted use, distribution, and reproduction in any medium, provided the original work is properly cited.

\section{INTRODUCTION}

Advances in miniaturization, electronics, and communications have made the use of sensor networks a popular choice for providing surveillance coverage in diverse application areas. Much of the current emphasis is on improved detection, classification, and localization of a single point in the surveillance region. However, recently, the use of a set of sensors that are geometrically distributed over a large area has been proposed as a cost-effective approach for tracking moving targets through surveillance regions (see, e.g., $[1-3]$ ). When designing these distributed sensor systems, the placement of the sensors within the field becomes a critical component of the design. Parametric representations of system performance goals, in terms of field parameters, provide an ability to appropriately consider trade-offs in the system design.

It has been shown by Cox [4] that beneficial detection performance can be obtained by sparsely distributed sensor networks when a multisensor detection strategy is employed in conjunction with a simple consistency check against expected target kinematics (i.e., a track-before-detect search procedure). By exploiting this kinematic check, these methods have been shown [5] to be robust against false alarms. This feature of track-before-detect strategies has been used to generate simple tracking procedures [6] that are robust against false alarms and require minimal between-sensor processing. The track-before-detect construct for distributed sensor networks is based on the ability of the collaborative effort of fixed, but distributed, sensors to report detections (over a network) to some higher-level system, where, then, a system-level detection decision is made based on a track estimate derived from the multiple detection reports. This higher-level system has the added benefit of effectively "weeding-out" false alarms that are inconsistent across the network; this benefit is one of the driving forces behind the employment of distributed sensor fields in harsh environments where communications capability between sensors is severely constrained. Other approaches to tracking targets with simple kinematics using sensor networks revolve around either a search theory perspective [7] or computationally efficient enumeration and filtering of potential tracks [8].

In a previous work, Wettergren [9] describes a design procedure for trading-off search coverage and false search when using a track-before-detect search strategy in a simple distributed sensor field. One of the objectives of this strategy is to maximize what is called the probability of successful search, defined as the probability of getting at least $k$ detections in a field of $N$ sensors. This probability is a function of many variables, any one of which may be random, and which include target course and speed, target location at some specified reference time, the locations of the sensors in the field, the range sensitivities and detection probabilities for the sensors, and the duration of the search. Prior 
studies [10] have shown how probabilistic modeling of target motion affects the efforts of a single searcher looking for the uncertain moving target. In this paper, we examine a related aspect of the track-before-detect search strategy for distributed sensor networks; namely, the localization accuracy of the search. Localization accuracy is defined in terms of the area of intersection of the sensor spatial-temporal coverage regions as seen from the target frame of reference. From this perspective, it is the target that is fixed, and the sensors that move with constant velocity (in the opposite direction). We show that, given $k$ detections, the target is detected by a set of $k$ sensors if and only if in the target coordinate system, the target is in the area of intersection of the coverage regions of these sensors. This area of intersection provides a measure (both graphical and quantifiable) of the expected area of uncertainty of the target location at a fixed point in time-even when the multiple sensor detections are not simultaneously obtained. The availability of a rapid assessment of expected localization accuracy in terms of sensor and target characteristics creates an invaluable design tool for proper positioning of sensors within the field.

This paper develops a set of calculations to determine the amount of expected localization accuracy that is attributable to the kinematic basis of track-before-detect methods. Under the assumption that individual sensor nodes report detections within some predictable range at a predictable accuracy, we build a simple model of track-before-detect systemlevel performance for a generic distributed sensor network. While this performance is not meant to be representative of any particular sensor system, it illustrates the impact of target kinematics on track-before-detect as a function of sensor positions.

The remainder of this paper is organized as follows. In Section 2, we describe sensor coverage in both sensor and target coordinate systems, define the area of intersection of these coverage regions given $k$ detections, and derive an expression for this area in terms of sensor and target variables. We then compute the expected area of intersection, and the variance of this area, for sensors distributed randomly according to a fixed and known, but arbitrary, distribution function. In Section 3, these results are used to calculate the expected value and variance of the area of intersection, given 1, 2, 3, or more detections on a target passing through the sensor field. Section 4 includes examples that verify experimentally the analytical results of Sections 2 and 3. These examples include a uniformly distributed sensor field, a sensor "barrier" consisting of sensors distributed uniformly in $x$ and normally in $y$, and, finally, an arbitrarily distributed sensor field. We conclude in Section 5 with a summary of our findings, and some suggestions for further study.

\section{INTERSECTION OF SENSOR SPATIAL-TEMPORAL COVERAGE REGIONS}

We consider the problem of a set of $N$ fixed identical sensors deployed to search for a single moving target using a trackbefore-detect search strategy. We limit our exposition to the discussion of a single target; the extension to multiple targets is discussed in the conclusion. Let $S \subseteq \mathbb{R}^{2}$ denote the region

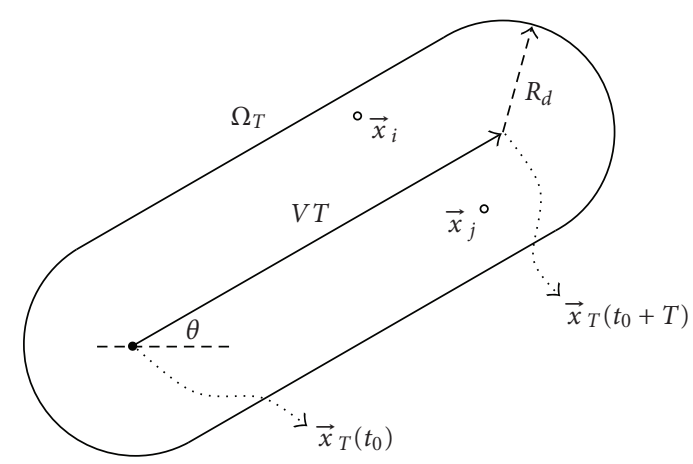

FIGURE 1: Detection region $\Omega_{T}$ in sensor coordinate system. Sensors $i$ and $j$ are in the detection region.

to be searched over the time interval $t_{0} \leq t \leq t_{0}+T$ (henceforth referred to as the search interval), and let $\vec{x}_{T}(t)$ denote the location of the target at time $t$. We assume that the target remains in the search region $S$ and moves with constant speed $V$ in a fixed direction $\theta$ throughout the search interval. The target track over the search interval is then given by

$$
\vec{x}_{T}(t)=\vec{x}_{T}\left(t_{0}\right)+\left(t-t_{0}\right) V(\cos \theta, \sin \theta)
$$

where we recall that $t$ parameterizes the search interval $t_{0} \leq$ $t \leq t_{0}+T$. Let $\vec{x}_{i}, i=1, \ldots, N$ denote the locations in $S$ of $N$ fixed sensors. We assume the sensors all have identical finite detection range $R_{d}$ and known probability of detection $P_{d}$. A target detection is defined to occur on sensor $i$ during the search interval with probability $P_{d}$ if and only if the target passes within a distance $R_{d}$ of the sensor (during that interval). Define the region $\Omega_{T}$ as

$$
\Omega_{T}=\left\{\vec{x} \in \mathbb{R}^{2}:\left\|\vec{x}-\vec{x}_{T}(t)\right\| \leq R_{d}, t_{0} \leq t \leq t_{0}+T\right\},
$$

where $\|\cdot\|$ denotes Euclidean distance. Hence, if sensor $i$ detects the target during the search interval, then $\vec{x}_{i} \in \Omega_{T}$. Moreover, if $k$ sensors detect the target during the search interval, then $\vec{x}_{i_{1}}, \ldots, \vec{x}_{i_{k}} \in \Omega_{T}$ for some subset $\left\{i_{1}, \ldots, i_{k}\right\}$ of $\{1, \ldots, N\}$. The region $\Omega_{T}$, referred to as a "target pill" in [9] because of its shape, is depicted in Figure 1. This region is the spatial-temporal coverage, or detection, region for the target.

A natural measure of localization accuracy is the area of uncertainty, which identifies a region of the search space $S$ where the target is located. Often, the area of uncertainty is presented as a collection of closed sets, where each member of the collection identifies a region of $S$ where the target is located with a certain probability. The area of uncertainty presented in this paper is a single connected closed subset of $S$ that contains the target with a probability one.

The search coverage region $\Omega_{T}$ in (2) is defined with respect to a sensor-referenced coordinate system, for which the area of uncertainty lacks a simple geometrical description. However, considering the target-referenced coordinate system (in which the target is fixed and the sensors move with constant speed in the opposite direction) provides a mechanism for examining the area of uncertainty over the multiple (nonsimultaneous) sensor detections in a geometrically intuitive manner, as described below. In this target frame of 


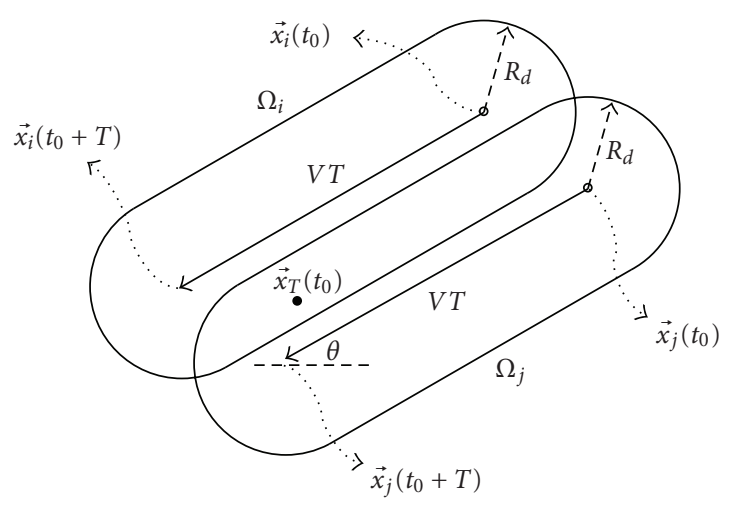

Figure 2: Detection regions $\Omega_{i}$ and $\Omega_{j}$ in target coordinate system. At time $t_{0}$, the target location $\vec{x}_{T}\left(t_{0}\right)$ is in the intersection of the detection regions for sensors $i$ and $j$.

reference, the target is fixed and the sensors move with speed $V$ in direction $\theta+\pi$. The track of sensor $i$ in the target coordinate system over the search interval is then given by

$$
\begin{aligned}
\vec{x}_{i}(t) & =\vec{x}_{i}\left(t_{0}\right)+\left(t-t_{0}\right) V(\cos (\theta+\pi), \sin (\theta+\pi)) \\
& =\vec{x}_{i}\left(t_{0}\right)-\left(t-t_{0}\right) V(\cos \theta, \sin \theta) .
\end{aligned}
$$

Recall that in the sensor coordinate system, if sensor $i$ detects the target during the search interval, then the target passes within a distance $R_{d}$ of the sensor. Thus, in the target coordinate system, if the target is detected by sensor $i$ during the search interval, then the sensor passes within $R_{d}$ of the target. For $i=1, \ldots, N$, let

$$
\Omega_{i}=\left\{\vec{x} \in S:\left\|\vec{x}-\vec{x}_{i}(t)\right\| \leq R_{d}, t_{0} \leq t \leq t_{0}+T\right\}
$$

represent the region of target detectability about sensor $i$. Thus, if sensor $i$ detects the target during the search interval $t_{0} \leq t \leq t_{0}+T$, then $\vec{x}_{T}\left(t_{0}\right) \in \Omega_{i}$. Furthermore, if the target is detected by $k$ sensors (e.g., sensors $i_{1}, \ldots, i_{k}$ ), then the target at time $t_{0}$ must lie in the intersection of the detection regions for these sensors, denoted $\Omega_{\text {int }}(k)$, that is,

$$
\vec{x}_{T}\left(t_{0}\right) \in \bigcap_{1 \leq j \leq k} \Omega_{i_{j}} \equiv \Omega_{\text {int }}(k) .
$$

This situation is depicted in Figure 2 for the case where the target is detected by two sensors, labeled $i$ and $j$. The region of intersection of the two "pills" $\Omega_{i}$ and $\Omega_{j}$ is the spatialtemporal detection region for the target in the target coordinate system.

Let $A_{\Omega}$ denote the area of the detection region $\Omega_{T}$. Since the transformation between the sensor and target reference frames is a pure translation, and since the sensor model is homogeneous in detection characteristics, it follows that $A_{\Omega}=\operatorname{area}\left(\Omega_{i}\right)$ for $i=1, \ldots, N$ as well. Given $k$ detections, let $A_{\text {int }}(k)$ denote the area of intersection of the $k$ detection regions in the target coordinates, that is, let $A_{\text {int }}(k)=$ area $\left(\Omega_{\text {int }}(k)\right)$. From the example in Figure 2, it is clear that $A_{\text {int }}(k)$ is a complicated function of the sensor locations, the sensor detection radius, the target initial location, course,

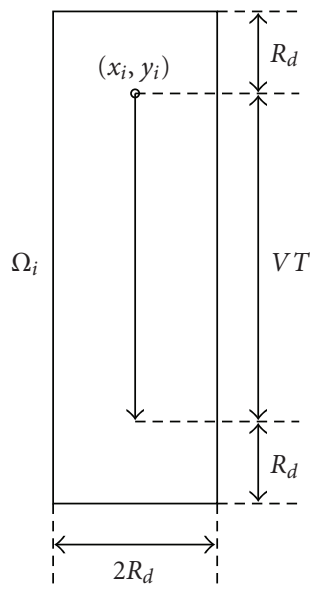

FIGURE 3: Rectangular coverage region for sensor $i$ in target coordinates.

and speed, and the length of the search interval. However, for $V T \gg R_{d}$, the region $\Omega_{i}$, with area $A_{\Omega}=\pi R_{d}^{2}+2 R_{d} V T$, is well approximated by the bounding rectangular region with dimensions $2 R_{d} \times\left(2 R_{d}+V T\right)$, and with area $4 R_{d}^{2}+2 R_{d} V T$. Recall that all sensors translate identically under the transformation to target coordinates, so all of the bounding rectangles for the different sensors are similarly aligned. Thus the intersection of any two of these overlapping rectangular detection regions is itself a rectangle with area greater than that of the intersection of the pills they bound. By induction, the intersection of any $k$ of these overlapping rectangles, $k \geq 2$, is a rectangle with area greater than that of the intersection of the $k$ corresponding pills. It follows that the area of intersection for the rectangular approximation to the pill-shaped detection regions is strictly greater than the area of the actual intersection and, hence, provides a strict upper bound on the area of uncertainty for track-before-detect systems under the circular "cookie-cutter" sensor model under consideration.

Throughout the sequel, let $\Omega_{i}$, the coverage region of sensor $i$ in the target frame of reference, be the rectangle of length $L_{y}=2 R_{d}+V T$ and width $L_{x}=2 R_{d}$. The rectangles are oriented such that the longer axis is parallel to the direction of target motion, taken here to be, without loss of generality, $\theta=\pi / 2$, corresponding to the $y$-axis. (Extensions to arbitrary target course $\theta$ are obtained by a simple rotation of coordinate axes.) The coverage region $\Omega_{i}$ is depicted in Figure 3. Note that the direction of sensor motion in the target coordinate system is $\theta+\pi=\pi / 2+\pi=3 \pi / 2$. This geometrical construction leads to $\Omega_{i}=\left\{(x, y) \in S: x_{i}-R_{d} \leq\right.$ $\left.x \leq x_{i}+R_{d}, y_{i}-V T-R_{d} \leq y \leq y_{i}+R_{d}\right\}$ for any sensor $i \in\{1, \ldots, N\}$ that detects the target. With these definitions, the following lemma provides a formula for the area of intersection of these rectangular detection regions in target coordinates given $k$ detections.

Lemma 1. Suppose there are $k \geq 1$ regions $\Omega_{i}$ with nonempty intersection corresponding to detections of a single target during the search interval. Without loss of generality, assume the sensors are labeled such that the detections occur on sensors 
$i \in\{1, \ldots, k\}$. Let $d_{x}(k)$ and $d_{y}(k)$ be defined as follows:

$$
\begin{aligned}
& d_{x}(k)=\max _{1 \leq i \leq k}\left(x_{i}\right)-\min _{1 \leq i \leq k}\left(x_{i}\right), \\
& d_{y}(k)=\max _{1 \leq i \leq k}\left(y_{i}\right)-\min _{1 \leq i \leq k}\left(y_{i}\right) .
\end{aligned}
$$

Then $A_{\text {int }}(k)$, the area of the region of joint intersection $\Omega_{\text {int }}(k)$ (as defined in (5)), is given by

$$
A_{\text {int }}(k)=\left(L_{x}-d_{x}(k)\right)\left(L_{y}-d_{y}(k)\right) .
$$

Proof. Take any point $p=(u, v)$ in $\mathbb{R}^{2}$. Then $p \in \Omega_{\text {int }}(k)$ if and only if

$$
\begin{gathered}
x_{i}-R_{d} \leq u \leq x_{i}+R_{d}, \\
y_{i}-V T-R_{d} \leq v \leq y_{i}+R_{d},
\end{gathered}
$$

for $i=1, \ldots, k$. These inequalities hold if and only if

$$
\begin{gathered}
\max _{1 \leq i \leq k}\left(x_{i}\right)-R_{d} \leq u \leq \min _{1 \leq i \leq k}\left(x_{i}\right)+R_{d}, \\
\max _{1 \leq i \leq k}\left(y_{i}\right)-V T-R_{d} \leq v \leq \min _{1 \leq i \leq k}\left(y_{i}\right)+R_{d} .
\end{gathered}
$$

Since the point $p=(u, v)$ in $\mathbb{R}^{2}$ is arbitrary, it follows that

$$
\begin{aligned}
\Omega_{\text {int }}(k)= & {\left[\max _{1 \leq i \leq k}\left(x_{i}\right)-R_{d}, \min _{1 \leq i \leq k}\left(x_{i}\right)+R_{d}\right] } \\
& \times\left[\max _{1 \leq i \leq k}\left(y_{i}\right)-V T-R_{d}, \min _{1 \leq i \leq k}\left(y_{i}\right)+R_{d}\right] .
\end{aligned}
$$

Now,

$$
\begin{aligned}
& \min _{1 \leq i \leq k}\left(x_{i}\right)+R_{d}-\left(\max _{1 \leq i \leq k}\left(x_{i}\right)-R_{d}\right) \\
& =\min _{1 \leq i \leq k}\left(x_{i}\right)-\max _{1 \leq i \leq k}\left(x_{i}\right)+2 R_{d}=L_{x}-d_{x}(k) .
\end{aligned}
$$

Likewise,

$$
\begin{aligned}
& \min _{1 \leq i \leq k}\left(y_{i}\right)+R_{d}-\left(\max _{1 \leq i \leq k}\left(y_{i}\right)-V T-R_{d}\right) \\
& =\min _{1 \leq i \leq k}\left(y_{i}\right)-\max _{1 \leq i \leq k}\left(y_{i}\right)+2 R_{d}+V T=L_{y}-d_{y}(k) .
\end{aligned}
$$

Thus the area $A_{\text {int }}(k)$ of the intersection $\Omega_{\text {int }}(k)$ is equal to $\left(L_{x}-d_{x}(k)\right)\left(L_{y}-d_{y}(k)\right)$. Note that for $k=1, d_{x}(1)=d_{y}(1)=$ 0 , and $A_{\text {int }}(1)=L_{x} L_{y}=\left(2 R_{d}\right)\left(2 R_{d}+V T\right)=A_{\Omega}$, as expected.

This lemma explicitly shows that the region of potential target locations for $k$ detections, $\Omega_{\text {int }}(k)$, and its area, $A_{\text {int }}(k)$, are functions of the sensor detection range $R_{d}$, the sensor locations $\vec{x}_{1}, \ldots, \vec{x}_{k}$, the target speed $V$, and the length $T$ of the search interval. Implicitly, $\Omega_{\text {int }}(k)$ and $A_{\text {int }}(k)$ are also functions of initial target location $\vec{x}_{T}\left(t_{0}\right)$, as the particular $k$-subset of $N$ sensors that detect the target obviously depends on the location of the target in the search space $S$. In general, any one of these variables may be random. This paper is concerned with the statistics of $A_{\text {int }}(k)$ as a function of $\vec{x}_{T}\left(t_{0}\right)$ when $R_{d}, V$, and $T$ are fixed and known, and the sensor locations $\vec{x}_{1}, \ldots, \vec{x}_{N}$ are distributed randomly in $S$ according to a fixed and known, but arbitrary, distribution function. The explicit computation of the expected value and variance of $A_{\text {int }}(k)$ in terms of $R_{d}, V, T, \vec{x}_{1}, \ldots, \vec{x}_{k}$, and $\vec{x}_{T}\left(t_{0}\right)$ provides a means for representing localization accuracy of track-before-detect search strategies in terms of these important distributed sensor system design parameters.

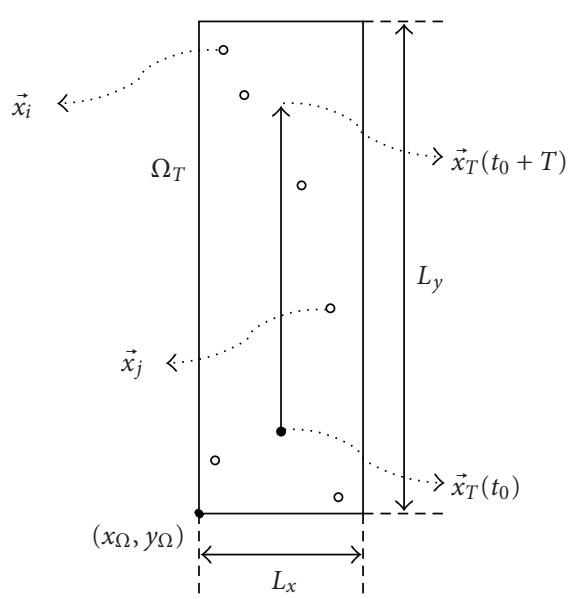

Figure 4: Rectangular detection region in sensor coordinates.

\subsection{Expected value of $A_{\text {int }}(k)$}

Suppose there are $k \geq 1$ detections on sensors $i=1, \ldots, k$. Since these sensors detect the target during the search inter$\mathrm{val}$, then it must be the case that, in the sensor frame of reference, $\vec{x}_{1}, \ldots, \vec{x}_{k} \in \Omega_{T}$. This situation is depicted in Figure 4, where only sensors $i$ and $j, 1 \leq i<j \leq k$, are explicitly labeled. Let $x_{(1)}, \ldots, x_{(k)}$ and $y_{(1)}, \ldots, y_{(k)}$ denote the order statistics [11] associated with the $x$ and $y$ coordinates, respectively, of the $k$ sensor locations. Lemma 1 gives the area of intersection $A_{\text {int }}(k)$ of the detection region $\Omega_{\text {int }}(k)$ as a function of the range (the maximum value minus the minimum value) of the order statistics $x_{(1)}, \ldots, x_{(k)}$ and $y_{(1)}, \ldots, y_{(k)}$, that is, $d_{x}(k)=x_{(k)}-x_{(1)}$ and $d_{y}(k)=y_{(k)}-y_{(1)}$. We use known results on the range of order statistics [11] to compute the expected value and variance of the area of intersection $A_{\text {int }}(k)$.

From Lemma 1, the area of intersection of the detection regions $\Omega_{1}, \ldots, \Omega_{k}$ is given by

$$
A_{\text {int }}(k)=A_{\Omega}\left(1-\frac{d_{x}(k)}{L_{x}}\right)\left(1-\frac{d_{y}(k)}{L_{y}}\right),
$$

where $A_{\Omega}=L_{x} L_{y}$ is the area of the coverage region $\Omega_{i}$ of a single sensor. If the sensor locations are distributed independently in $x$ and $y$, then the expected value of $A_{\text {int }}(k)$ is given by

$$
E\left(A_{\text {int }}(k)\right)=A_{\Omega}\left(1-\frac{E\left(d_{x}(k)\right)}{L_{x}}\right)\left(1-\frac{E\left(d_{y}(k)\right)}{L_{y}}\right) .
$$

The sensor locations within the search region $S$ are assumed to be random, with a fixed and known distribution function. Let $F(x, y)$ represent the distribution function corresponding to the random locations of the sensors. The corresponding density function $f(x, y)$ is given by $f=F^{\prime}$. Furthermore, let $f_{X}$ and $f_{Y}$ represent the marginal density functions of the sensor locations in the $x$ and $y$ coordinates, respectively, with associated distribution functions $F_{X}$ and $F_{Y}$. Since the ranges $d_{x}(k)$ and $d_{y}(k)$ depend on the locations of the detecting sensors, the expected values $E\left(d_{x}(k)\right)$ and $E\left(d_{y}(k)\right)$ clearly depend on these distribution functions. In particular, from the 
theory of order statistics (see Stuart and Ord [11, page 495]), the expected value of $d_{x}(k)$ is given by

$$
E\left(d_{x}(k)\right)=\int_{x_{\Omega}}^{x_{\Omega}+L_{x}}\left\{1-\left[F_{X \mid \Omega_{T}}(x)\right]^{k}-\left[1-F_{X \mid \Omega_{T}}(x)\right]^{k}\right\} d x,
$$

where $F_{X \mid \Omega_{T}}$ and $F_{Y \mid \Omega_{T}}$ represent the conditional distribution functions for $F_{X}$ and $F_{Y}$, respectively, conditioned on the detection region $\Omega_{T}$. Equation (15) is derived in [11] by substituting the well-known density functions for the minimum and maximum order statistics $x_{(1)}$ and $x_{(k)}$ into the identity $E\left(d_{x}(k)\right)=E\left(x_{(k)}\right)-E\left(x_{(1)}\right)$, and using integration by parts to simplify the resulting expression. A similar expression to (15) holds for the expected value of the range $d_{y}(k)$.

The conditional distribution function $F_{X \mid \Omega_{T}}$ is given by

$$
F_{X \mid \Omega_{T}}(x)= \begin{cases}0, & x<x_{\Omega}, \\ \int_{x_{\Omega}}^{x} f_{X \mid \Omega_{T}}(\xi) d \xi, & x_{\Omega} \leq x \leq x_{\Omega}+L_{x}, \\ 1, & x>x_{\Omega}+L_{x},\end{cases}
$$

where the point $\left(x_{\Omega}, y_{\Omega}\right)$ denotes the lower left-hand corner of $\Omega_{T}$ (see Figure 4 ), and

$$
f_{X \mid \Omega_{T}}(x)=\frac{f_{X}(x)}{\int_{x_{\Omega}}^{x_{\Omega}+L_{x}} f_{X}(\xi) d \xi}, \quad x_{\Omega} \leq x \leq x_{\Omega}+L_{x},
$$

and similarly for $F_{Y \mid \Omega_{T}}$ and $f_{Y \mid \Omega_{T}}$. Thus, for a known distribution on the sensor locations, the expected value of the area of the target location region is computed from (14), (15), and (16) (including the corresponding expressions for $E\left(d_{y}(k)\right.$ ) and $\left.F_{Y \mid \Omega_{T}}(y)\right)$.

If the sensor locations are not distributed independently in $x$ and $y$, the expectation operator does not, in general, distribute across terms in $A_{\text {int }}(k)$ by (14). However, in practice, we expect long, narrow detection regions, which is the case for $V T \gg R_{d}$. For a target with course $\theta=\pi / 2$, this translates to a detection region $\Omega_{T}$ with $L_{y} \gg L_{x}$. Also, for a search region $S$ much larger than the detection region $\Omega_{T}$, we expect the variation in $f_{X \mid \Omega_{T}}$ over the interval $x_{\Omega} \leq x \leq x_{\Omega}+L_{x}$ to be small for all values of $x_{\Omega}$. With these assumptions, the sensor $x$ and $y$ locations are distributed approximately independently in $\Omega_{T}$, with sensor $x$ location approximately uniformly distributed in this region, yielding

$$
f_{X \mid \Omega_{T}}(x)= \begin{cases}\frac{1}{L_{x}}, & x_{\Omega} \leq x \leq x_{\Omega}+L_{x}, \\ 0, & \text { otherwise }\end{cases}
$$

which greatly simplifies the evaluation of (15).

\subsection{Variance of $A_{\text {int }}(k)$}

The variance of the area of intersection of the detection regions $\Omega_{1}, \ldots, \Omega_{k}$, denoted $\operatorname{var}\left(A_{\text {int }}(k)\right)$, is given by

$$
\begin{aligned}
\operatorname{var}\left(A_{\text {int }}(k)\right) & =E\left(\left(A_{\text {int }}(k)-E\left(A_{\text {int }}(k)\right)\right)^{2}\right) \\
& =E\left(\left(A_{\text {int }}(k)\right)^{2}\right)-\left(E\left(A_{\text {int }}(k)\right)\right)^{2},
\end{aligned}
$$

where $E\left(A_{\text {int }}(k)\right)$ is given by (14). As in the previous section, the sensor $x$ and $y$ locations are approximately independent (within the local region $\Omega_{T}$ ), leading to

$$
E\left(\left(A_{\text {int }}(k)\right)^{2}\right)=A_{\Omega}^{2} E\left(\left(1-\frac{d_{x}(k)}{L_{x}}\right)^{2}\right) E\left(\left(1-\frac{d_{y}(k)}{L_{y}}\right)^{2}\right),
$$

where

$$
\begin{aligned}
& E\left(\left(1-\frac{d_{x}(k)}{L_{x}}\right)^{2}\right) \\
& \quad=1-2 \frac{E\left(d_{x}(k)\right)}{L_{x}}+\frac{E\left(d_{x}^{2}(k)\right)}{L_{x}^{2}} \\
& =1-2 \frac{E\left(d_{x}(k)\right)}{L_{x}}+\frac{\left(E\left(d_{x}(k)\right)\right)^{2}+\operatorname{var}\left(d_{x}(k)\right)}{L_{x}^{2}} \\
& =\left(1-\frac{E\left(d_{x}(k)\right)}{L_{x}}\right)^{2}+\frac{\operatorname{var}\left(d_{x}(k)\right)}{L_{x}^{2}},
\end{aligned}
$$

and similarly for the $d_{y}(k)$ term. The expected value of the range $d_{x}(k)$ is given by (15); a similar expression gives the expected value of the range $d_{y}(k)$. The variances of the ranges $d_{x}(k)$ and $d_{y}(k)$ are found using known results on order statistics. From [11, page 495],

$$
\begin{aligned}
\operatorname{var} & \left(d_{x}(k)\right) \\
= & 2 \int_{x_{\Omega}}^{x_{\Omega}+L_{x}} \int_{x_{\Omega}}^{x_{(n)}}\left\{1-\left[F_{X \mid \Omega_{T}}\left(x_{(n)}\right)\right]^{k}-\left[1-F_{X \mid \Omega_{T}}\left(x_{(1)}\right)\right]^{k}\right. \\
& \left.+\left[F_{X \mid \Omega_{T}}\left(x_{(n)}\right)-F_{X \mid \Omega_{T}}\left(x_{(1)}\right)\right]^{k}\right\} d x_{(1)} d x_{(n)-\left(E\left(d_{x}(k)\right)\right)^{2}}
\end{aligned}
$$

for $k \geq 1$, and similarly for $\operatorname{var}\left(d_{y}(k)\right)$. The change of variables $u=x_{(k)}, v=\left(x_{(1)}-x_{\Omega}\right) /\left(x_{(k)}-x_{\Omega}\right)$ replaces the iterated integral in (22) by one with constant limits of integration, yielding

$$
\begin{aligned}
& \operatorname{var}\left(d_{x}(k)\right) \\
& =2 \int_{x_{\Omega}}^{x_{\Omega}+L_{x}} \int_{0}^{1}\left\{1-\left[F_{X \mid \Omega_{T}}(u)\right]^{k}\right. \\
& -\left[1-F_{x \mid \Omega_{T}}\left((1-v) x_{\Omega}+u v\right)\right]^{k} \\
& \left.+\left[F_{X \mid \Omega_{T}}(u)-F_{X \mid \Omega_{T}}\left((1-v) x_{\Omega}+u v\right)\right]^{k}\right\} \\
& \times\left(u-x_{\Omega}\right) d v d u \\
& -\left(E\left(d_{x}(k)\right)\right)^{2}
\end{aligned}
$$

for $k \geq 1$, and similarly for $\operatorname{var}\left(d_{y}(k)\right)$. These latter expressions for the variances of the ranges $d_{x}(k)$ and $d_{y}(k)$ are more amenable to numerical evaluation, and are used for the examples in Section 4.

Observe that for $k=1,(22)$ and (23) yield $\operatorname{var}\left(d_{x}(1)\right)=$ 0 . Substituting this result into $(21)$ gives $E\left(\left(1-d_{x}(1) / L_{x}\right)^{2}\right)=$ 1 , and substituting this result into $(20)$ gives $E\left(\left(A_{\text {int }}(1)\right)^{2}\right)=$ $A_{\Omega}^{2}$. It then follows from (19) that $\operatorname{var}\left(A_{\text {int }}(1)\right)=0$. This result is expected, since given a singledetection from sensor $i$, 
the area of uncertainty is precisely the area of the detection region $\Omega_{i}$ (equivalently, the detection region $\Omega_{T}$ ).

\subsection{Uniform sensor distribution}

Given the general expressions for the expected value and variance of $A_{\text {int }}(k)$, we now examine the special cases when sensor location is distributed according to the uniform distribution in one or both coordinates. In the latter case, the expected value and variance of $A_{\text {int }}(k)$ take simple closed forms. As described in Section 2.1, the case of sensors uniformly distributed in $x$ is a general assumption considered in practice. This assumption leads to simplification based on the following lemma.

Lemma 2. Suppose the sensor $x$ locations are distributed uniform $\left(x_{\Omega}, x_{\Omega}+L_{x}\right)$ in $\Omega_{T}$. Then $d_{x}(k) / L_{x}$ has mean and variance given by

$$
\begin{aligned}
E\left(\frac{d_{x}(k)}{L_{x}}\right) & =\frac{E\left(d_{x}(k)\right)}{L_{x}}=\frac{k-1}{k+1}, \\
\operatorname{var}\left(\frac{d_{x}(k)}{L_{x}}\right) & =\frac{\operatorname{var}\left(d_{x}(k)\right)}{L_{x}^{2}}=\frac{2(k-1)}{(k+1)^{2}(k+2)},
\end{aligned}
$$

respectively. Moreover, $d_{x}(k) / L_{x}$ is distributed beta $(k-1,2)$.

The detailed proof of Lemma 2 is given in the appendix. Incidentally, this lemma holds equally for sensors with $y$ locations distributed uniform $\left(y_{\Omega}, y_{\Omega}+L_{y}\right)$. This observation leads to the following theorem.

Theorem 1. If sensor $x$ and $y$ locations are distributed independently and uniformly in $\Omega_{T}$, then

$$
\begin{aligned}
E\left(A_{\text {int }}(k)\right) & =\frac{4 A_{\Omega}}{(k+1)^{2}}, \\
\operatorname{var}\left(A_{\text {int }}(k)\right) & =\frac{4\left(5 k^{2}+2 k-7\right) A_{\Omega}^{2}}{(k+1)^{4}(k+2)^{2}} .
\end{aligned}
$$

Proof. Since the sensors are assumed distributed uniformly in $x$ and $y$, Lemma 2 gives

$$
\frac{E\left(d_{x}(k)\right)}{L_{x}}=\frac{E\left(d_{y}(k)\right)}{L_{y}}=\frac{k-1}{k+1} .
$$

Substituting this result into (14) yields

$$
E\left(A_{\text {int }}(k)\right)=A_{\Omega}\left(1-\frac{k-1}{k+1}\right)^{2}=\frac{4 A_{\Omega}}{(k+1)^{2}} .
$$

Since $d_{x}(k)$ and $d_{y}(k)$ are independent and identically distributed, (19), (20), and (21) yield

$$
\begin{aligned}
& \operatorname{var}\left(A_{\text {int }}(k)\right) \\
& =A_{\Omega}^{2}\left[\left(1-\frac{E\left(d_{x}(k)\right)}{L_{x}}\right)^{2}+\frac{\operatorname{var}\left(d_{x}(k)\right)}{L_{x}^{2}}\right]^{2}-\left(E\left(A_{\text {int }}(k)\right)\right)^{2} .
\end{aligned}
$$

Substituting the expressions for $E\left(d_{x}(k)\right)$ and $\operatorname{var}\left(d_{x}(k)\right)$ as given in Lemma 2, along with (25) for $E\left(A_{\text {int }}(k)\right)$ gives

$$
\begin{aligned}
\operatorname{var}\left(A_{\text {int }}(k)\right) & =\frac{36 A_{\Omega}^{2}}{(k+1)^{2}(k+2)^{2}}-\frac{16 A_{\Omega}^{2}}{(k+1)^{4}} \\
& =\frac{4\left(5 k^{2}+2 k-7\right) A_{\Omega}^{2}}{(k+1)^{4}(k+2)^{2}} .
\end{aligned}
$$

We note that Theorem 1 shows that the localization accuracy depends only upon the area of the detection region $\Omega_{T}$ and the number of detections $k$; it does not explicitly depend on the number of sensors nor sensor density. However, there is an implicit dependence on these quantities since obtaining $k$ detections requires a minimal number of sensors, as shown in [9].

\section{DISTRIBUTION OF $A_{\text {int }}(k)$ GIVEN $k \geq \ell$}

The quantities $E\left(A_{\text {int }}(k)\right)$ and $\operatorname{var}\left(A_{\text {int }}(k)\right)$ represent the expected value and variance, respectively, of the area of the uncertainty region $\Omega_{\text {int }}(k)$, given $k$ detections. When a sensor field is deployed and operating, the number of detections $k$ is itself a random variable and, like $A_{\text {int }}(k)$, is a function of the sensor locations and detection characteristics, the target kinematics, and the search interval. The distribution function for $k$ as a function of these variables is given by Wettergren in [9]. This probability distribution is used to obtain the expected value and variance of the area of uncertainty given at least $\ell$ detections, that is, given $k \geq \ell$.

Let $K$ denote the random variable associated with the observed number of detections $k$. Then the probability of getting $k$ detections is denoted $P(K=k)$, the probability of getting at least one detection is denoted $P(K \geq 1)$, and so on. Incidentally, the probability of getting at least $k$ detections, $P(K \geq k)$, is referred to in [9] as the (system level) probability of successful search, and is also denoted by $P_{\mathrm{SS}}(k)$. A successful search is defined in [9] as the event of obtaining at least $k$ detections for some prescribed value of $k$; this event occurs with probability $P_{\mathrm{SS}}(k)$.

The probability of getting exactly $k$ detections, as well as the system level probability of successful search $P_{\mathrm{SS}}(k)$, depends on the sensor level probability of successful search, denoted $p$ in [9]. This probability is defined as

$$
p=1-\exp \left(-P_{d} \varphi\right)
$$

where $P_{d}$ is the (a priori) sensor probability of detection, and $\varphi$ is the probability of finding a sensor in the spatial-temporal target detection region $\Omega_{T}$, that is,

$$
\varphi=\int_{\Omega_{T}} f(\vec{x}) d \vec{x}
$$


where $f$ is the sensor location density function. The event of getting exactly $k$ detections is defined in terms of the outcome of $N$ independent Bernoulli trials ( $N$ being the total number of sensors), with success probability $p$, as given by (31), and failure probability $1-p$. Then the resulting distribution function for the number of observed detections $k$ is given by the binomial distribution

$$
P(K=k)=\left(\begin{array}{l}
N \\
k
\end{array}\right) p^{k}(1-p)^{N-k}, \quad k=0,1, \ldots, N .
$$

The corresponding conditional distribution function $P(K=$ $k \mid K \geq \ell)$ is given by

$$
P(K=k \mid K \geq \ell)= \begin{cases}0, & \text { if } k<\ell, \\ \frac{P(K=k)}{1-\sum_{0 \leq i<\ell} P(K=i)}, & \text { if } k \geq \ell .\end{cases}
$$

Let $E\left(A_{\text {int }} \mid \ell\right)=E\left(A_{\text {int }}(K) \mid K \geq \ell\right)$ denote the expected value of $A_{\text {int }}(k)$ given at least $\ell$ detections. Likewise, let $\operatorname{var}\left(A_{\text {int }} \mid \ell\right)=\operatorname{var}\left(A_{\text {int }}(K) \mid K \geq \ell\right)$ denote the variance of $A_{\text {int }}(k)$ given $k \geq \ell$. Then

$$
\begin{aligned}
E\left(A_{\text {int }} \mid \ell\right) & =\sum_{0 \leq k \leq N} \mathrm{E}\left(A_{\text {int }}(k)\right) P(K=k \mid K \geq \ell), \\
\operatorname{var}\left(A_{\text {int }} \mid \ell\right) & =\sum_{0 \leq k \leq N} \operatorname{var}\left(A_{\text {int }}(k)\right) P(K=k \mid K \geq \ell),
\end{aligned}
$$

where $E\left(A_{\text {int }}(k)\right)$ and $\operatorname{var}\left(A_{\text {int }}(k)\right)$ are given, in general, by (14) and (19), respectively.

Finally, as pointed out by Wettergren in [9], binomial probabilities such as (33) and (34) are difficult to evaluate numerically for even moderate numbers of sensors because of the $N$ ! term in the binomial coefficient. However, the size of the detection region $\Omega_{T}$ is typically small compared to the size of the search space $S$ so that the probability $\varphi$ of finding a sensor in $\Omega_{T}$ is much less than one. Thus, for $P_{d}<1$, we have, from (31), that $p \approx 1-\left(1-P_{d} \varphi\right)=P_{d} \varphi$. Hence, for $\varphi \ll 1$, we conclude that $p \ll 1$. For $N \gg 1$, the DeMoivreLaplace theorem [12] provides an approximate evaluation of the binomial coefficient. In the case of $N \gg 1$ and $p \ll 1$, the distribution of Bernoulli trials is well-approximated by the limiting case of the Poisson theorem, yielding

$$
P(K=k)=\left(\begin{array}{l}
N \\
k
\end{array}\right) p^{k}(1-p)^{N-k} \approx \frac{(N p)^{k}}{k !} \exp (-N p),
$$

(see Feller [12, Chapter 6, Section 5]). As an example of the use of this approximation, substituting the approximation into expression (34) for the conditional probability of getting $k$ detections, having gotten at least one $(\ell=1)$ detection, gives

$$
\begin{aligned}
& P(K=k \mid K \geq 1) \\
& \approx \frac{1}{1-\left(1-P_{d} \varphi\right)^{N}} \frac{\left(N P_{d} \varphi\right)^{k}}{k !} \exp \left(-N P_{d} \varphi\right), \quad 1 \leq k \leq N,
\end{aligned}
$$

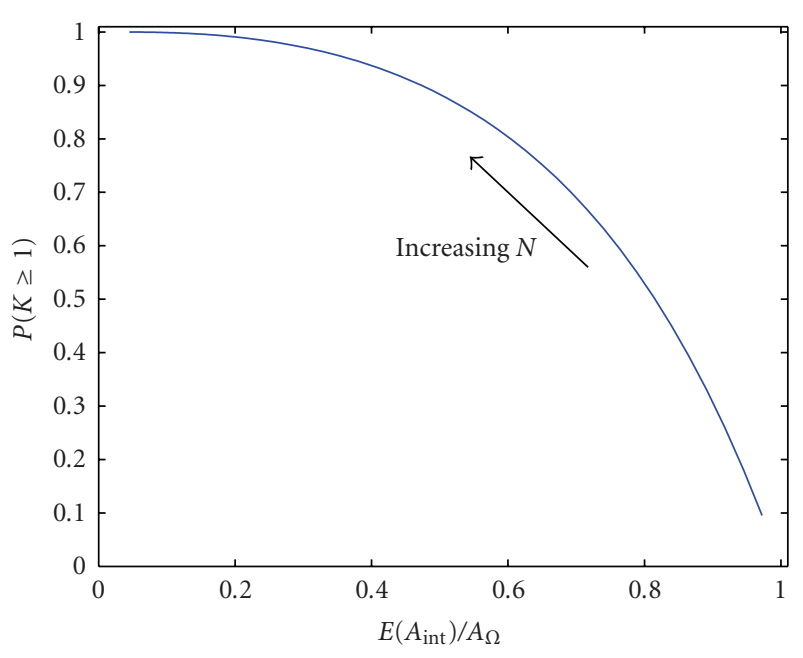

FIGURe 5: Probability of receiving a detection versus expected localization accuracy.

where the probability of receiving at least one detection is $P(K \geq 1)=1-\left(1-P_{d} \varphi\right)^{N}$.

In Figure 5, the probability of receiving at least one detection is plotted versus the expected value of the area of intersection for a set of sensors uniformly distributed over the search region. For convenience, the expected area of intersection is normalized by the single detection area $A_{\Omega}$. The curve in the figure is parameterized by the density of sensors in the search region (or, equivalently, the number of sensors $N)$. When there are very few sensors, the probability of detection is small, and when detection occurs, it is usually only a single sensor detection and thus the expected area of intersection corresponds to the detection region of a single sensor $\left(\right.$ since $\left.E\left(A_{\text {int }}(1)\right)=A_{\Omega}\right)$. Thus the normalized expected area of intersection approaches unity for small numbers of sensors. As the number of sensors increases, the likelihood of receiving more than one detection in the search interval increases, thus increasing the probability of at least one detection $(P(K \geq 1))$, as well as decreasing the expected area of intersection due to the reduction in the size of the intersection region with increasing numbers of detections (see (25)). The expected detection and localization performance of a distributed sensor field design can thus be set by carefully considering these relationships when determining the density of sensors to employ in the field.

\section{LOCALIZATION EXAMPLES}

In this section, we examine the localization accuracy of the track-before-detect search strategy described in [9] for a target with speed $V=1$ moving in direction $\theta=\pi / 2$ through a search space $S=[-10,10] \times[-10,10]$ covered by a field of $N=50$ sensors, each with detection range $R_{d}=1$, and over a search interval of duration $T=5$. In particular, the mean and variance of the area of uncertainty $A_{\text {int }}(k)$ given at least one detection are examined as functions of target location at the midpoint of the detection region $\Omega_{T}$. These example calculations are performed for three random sensor distributions: 
a uniform distribution, a barrier distribution, and an arbitrary distribution.

Note that for this example, the area $A_{\Omega}$ of the detection regions $\Omega_{T}$ and $\left\{\Omega_{i}\right\}_{1 \leq i \leq k}$ is equal to $4 R_{d}^{2}+2 R_{d} V T=4+$ $2 \cdot 5=14$. Then, for $k=1$, we have

$E\left(A_{\text {int }}(K) \mid K=1\right)=A_{\Omega}=14, \quad \operatorname{var}\left(A_{\text {int }}(K) \mid K=1\right)=0$.

Furthermore, in regions of $S$ for which the sensor location density function has little support, we have $P(K=k \mid K \geq$ $1) \approx 0$ for all $1<k \leq N$. In these regions, (35) imply

$$
E\left(A_{\text {int }} \mid 1\right) \approx A_{\Omega}=14, \quad \operatorname{var}\left(A_{\text {int }} \mid 1\right) \approx 0
$$

These observations are illustrated in the examples of Sections 4.2 and 4.3. The sensor location density functions for the examples in these sections have near zero support in large regions of the search space $S$.

\subsection{Uniform sensor field}

We first consider the 50 sensors distributed in $S$ according to the uniform distribution function, that is, the sensor $x$ and $y$ locations are independently and identically distributed uniform $(-10,10)$. Substituting the results of Theorem 1 into expressions (35), the expected value and variance of $A_{\text {int }}(k)$ given at least one detection are given by the following:

$$
\begin{aligned}
& E\left(A_{\text {int }} \mid 1\right) \\
&= \frac{4 A_{\Omega}}{1-\left(1-P_{d} \varphi\right)^{N}} \sum_{1 \leq k \leq N} \frac{\left(N P_{d} \varphi\right)^{k}}{(k+1)(k+1) !} \exp \left(-N P_{d} \varphi\right), \\
& \operatorname{var}\left(A_{\text {int }} \mid 1\right) \\
&=\frac{4 A_{\Omega}^{2}}{1-\left(1-P_{d} \varphi\right)^{N}} \sum_{1 \leq k \leq N} \frac{\left(5 k^{2}+2 k-7\right)\left(N P_{d} \varphi\right)^{k}}{(k+1)^{3}(k+2)(k+2) !} \\
& \quad \times \exp \left(-N P_{d} \varphi\right) .
\end{aligned}
$$

These analytical results are verified experimentally by estimating $E\left(A_{\text {int }} \mid 1\right)$ and $\operatorname{var}\left(A_{\text {int }} \mid 1\right)$ from a sequence of random draws of 50 sensors from the uniform distribution function on the search space $S$. In particular, consider the detection region $\Omega_{T}$ centered at the origin of $S$. For $m$ random draws of 50 sensors, let $m_{k}$ be the number of times $k$ sensors are in the region $\Omega_{T}$ for $k=0,1, \ldots, 50$. For the $i$ th draw out of $m$ draws, if the number of detections $k>0$, set $A_{i}(k)$ equal to $A_{\text {int }}(k)$, computed using expression (7). Given $m$ random draws, the probability of getting $k$ detections given $k \geq 1$ is estimated by

$$
\bar{P}(k)=\frac{m_{k} / m}{1-m_{0} / m}=\frac{m_{k}}{m-m_{0}},
$$

and the mean and variance of the area of intersection given $k$ detections are estimated by the sample statistics $\bar{A}_{\text {int }}(k)$ and $\bar{V}_{\text {int }}(k)$, respectively, as given by

$$
\begin{aligned}
& \bar{A}_{\text {int }}(k)=\frac{1}{m} \sum_{1 \leq i \leq m} A_{i}(k), \\
& \bar{V}_{\text {int }}(k)=\frac{1}{m} \sum_{1 \leq i \leq m}\left(A_{i}(k)-\bar{A}_{\text {int }}(k)\right)^{2} .
\end{aligned}
$$

The estimated mean and variance of $A_{\text {int }}(k)$ given at least one detection, denoted $\bar{A}_{\text {int }}$ and $\bar{V}_{\text {int }}$, respectively, are computed by combining these results, as in (35):

$$
\bar{A}_{\text {int }}=\sum_{1 \leq k \leq N} \bar{P}(k) \bar{A}_{\text {int }}(k), \quad \bar{V}_{\text {int }}=\sum_{1 \leq k \leq N} \bar{P}(k) \bar{V}_{\text {int }}(k) .
$$

Figures 6(a) and 6(b) show box plots of 300 values of $\bar{A}_{\text {int }}$ and $\bar{V}_{\text {int }}$, where each pair of values is estimated from $m=100$ and $m=1000$ samples of 50 sensors, respectively. The top and bottom lines of each box represent the upper and lower quartile values of the sample, and the line in-between these two lines represents the sample median; the dashed lines ("whiskers") extending from the top and bottom of each box represent the spread of the remaining sample, and any plus signs beyond the whiskers represent outliers. The true values $E\left(A_{\text {int }} \mid 1\right)=8.1540$ and $\operatorname{var}\left(A_{\text {int }} \mid 1\right)=4.6338$ for this example, computed using (40), are indicated in these plots by asterisks. Clearly, the uncertainty in our estimates of $E\left(A_{\text {int }} \mid 1\right)$ and $\operatorname{var}\left(A_{\text {int }} \mid 1\right)$ decreases with an increase in the number of 50-sensor samples, from 100 to 1000 , over the 300 experiments.

\subsection{Sensor barrier}

Now, consider a nonuniform sensor distribution in the search region $S$ in which the sensors are distributed in the $x$ and $y$ dimensions according to the uniform and normal distribution functions, respectively. Specifically, consider the sensor $x$ locations distributed independently uniform $(-10,10)$, and the sensor $y$ locations distributed independently normal $(\mu, \sigma)$ with mean $\mu=0$ and standard deviation $\sigma=2$. Contours of the joint density function $f_{X Y}$ are plotted in Figure 7, along with a sample of 50 sensors. This distribution forms a natural barrier against targets moving across the line $y=\mu$; hence, we refer to it as a barrier distribution.

The expected value and variance of the area of uncertainty $A_{\text {int }}$, given at least one detection, are found using the results of Sections 2.1, 2.2, and 3. These results require the conditional distribution functions $F_{X \mid \Omega_{T}}(x)$ and $F_{Y \mid \Omega_{T}}(y)$. For sensors distributed independently uniform $(-10,10)$ in the $x$ dimension, we have $f_{X \mid \Omega_{T}}(x)=1 / L_{x}$, which gives $F_{X \mid \Omega_{T}}(x)=\left(x-x_{\Omega}\right) / L_{x}$ for $x$ restricted to $\Omega_{T}$. Let $\phi$ denote the standard normal density function (with zero mean and standard deviation one), and let $\Phi$ denote its distribution function, so that, for $-\infty<t<\infty$,

$$
\begin{gathered}
\varphi(t)=\frac{1}{\sqrt{2 \pi}} \exp \left(-t^{2} / 2\right) \\
\Phi(t)=\int_{-\infty}^{t} \phi(\tau) d \tau=\frac{1}{2}(1+\operatorname{erf}(t / \sqrt{2})) .
\end{gathered}
$$




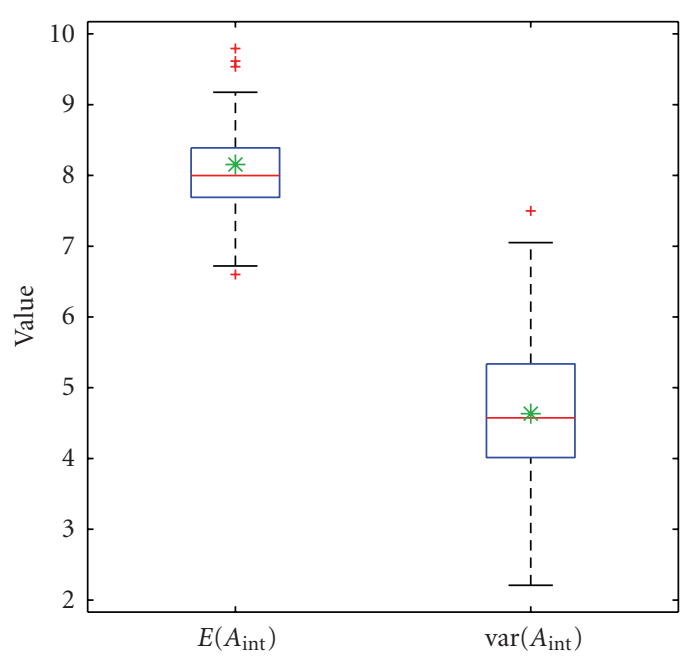

(a) Estimated from 100 50-sensor samples

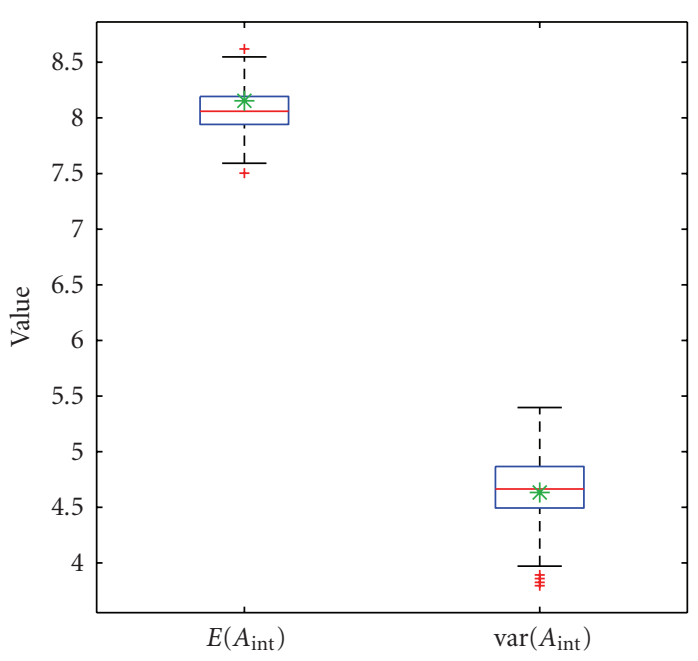

(b) Estimated from 1000 50-sensor samples

Figure 6: Box plots of 300 experimental values of $E\left(A_{\text {int }} \mid 1\right)$ and $\operatorname{var}\left(A_{\text {int }} \mid 1\right)$, each pair estimated from (a) 100 and (b) 1000 samples of 50 sensors. The asterisks indicate the analytical values $E\left(A_{\text {int }} \mid\right.$ $1)=8.1540$ and $\operatorname{var}\left(A_{\text {int }} \mid 1\right)=4.6338$ given by $(40)$, respectively.

It follows that, for sensors distributed independently $\operatorname{normal}(\mu, \sigma)$ in the $y$ dimension, we have, for $y$ restricted to $\Omega_{T}$,

$$
f_{Y \mid \Omega_{T}}(y)=\frac{1}{c \sigma} \phi\left(\frac{y-\mu}{\sigma}\right),
$$

with normalization constant $c$ given by

$$
c=\Phi\left(\frac{y_{\Omega}+L_{y}-\mu}{\sigma}\right)-\Phi\left(\frac{y_{\Omega}-\mu}{\sigma}\right) .
$$

Consequently, the conditional distribution function $F_{Y \mid \Omega_{T}}$ for this example is given by

$$
F_{Y \mid \Omega_{T}}(y)=\frac{1}{c}\left(\Phi\left(\frac{y-\mu}{\sigma}\right)-\Phi\left(\frac{y_{\Omega}-\mu}{\sigma}\right)\right) .
$$

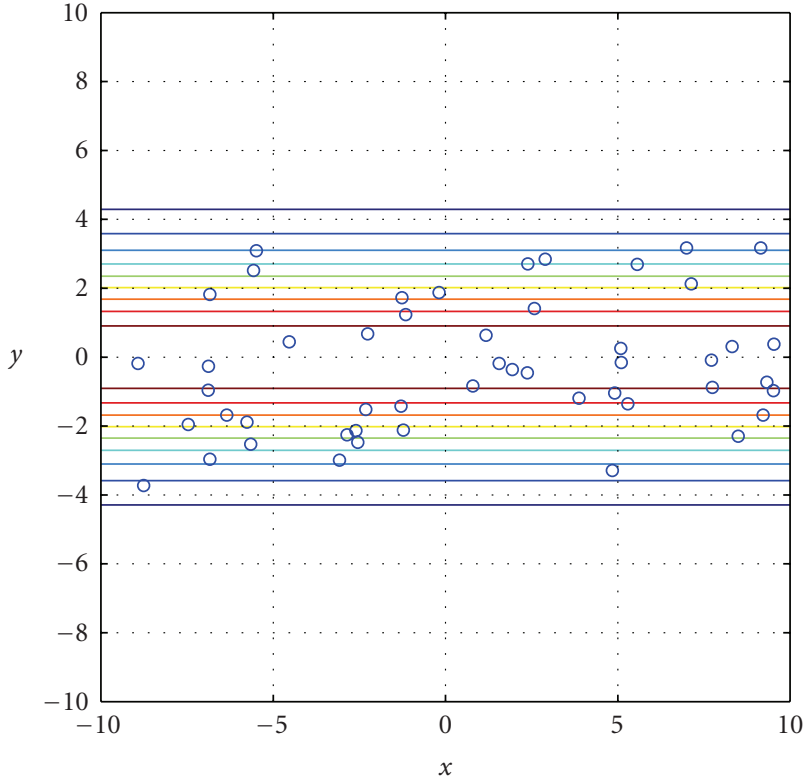

FIGURE 7: Sensor location density function for the barrier example, with $N=50$ sampled sensors.

Since the sensor locations are distributed independently in the $x$ and $y$ dimensions, and, moreover, uniformly in the $x$ dimension, the expected values and variances of $A_{\text {int }}(k)$ and $A_{\text {int }}$ are independent of sensor $x$ location. Figure 8 shows plots of $E\left(A_{\text {int }} \mid 1\right)$ (solid line) and $\operatorname{var}\left(A_{\text {int }} \mid 1\right.$ ) (dashed line) for the midpoint of the target track at $y=$ $-6.5,-5.5, \ldots, 5.5,6.5$. The endpoints -6.5 and 6.5 are chosen so that the bottom and top of the detection region $\Omega_{T}$ about the target track coincide with the bottom and top, respectively, of the search space $S$. The theoretical curves in Figure 8 are verified experimentally by estimating $E\left(A_{\text {int }} \mid 1\right)$ and $\operatorname{var}\left(A_{\text {int }} \mid 1\right)$ using the same approach as in the previous example. In this example, instead of estimating the sample statistics $\bar{A}_{\text {int }}$ and $\bar{V}_{\text {int }}$ for $m=100050$-sensor draws and for the detection region $\Omega_{T}$ centered at the origin of $S$, we compute these statistics for $m=100050$-sensor draws and for the sensor detection region $\Omega_{T}$ centered at $x=0$ and each of the locations $y=-6.5,-5.5, \ldots, 5.5,6.5$. For each of these 14 locations of the detection region $\Omega_{T}$ on the $y$-axis, 19 values of $\bar{A}_{\text {int }}$ and $\bar{V}_{\text {int }}$ are plotted in Figure 8 as circles and crosses, respectively. These estimates show good agreement with the theoretical curves.

The analytical and experimental results in Figure 8 show some interesting trends. That the expected area of intersection, or area of uncertainty, should decrease monotonically as the target enters the sensor barrier, and then increase at the opposite rate as the target leaves the barrier, is intuitively obvious, given the symmetry of this example. Few, if any, detections are expected in the tails of the barrier; it follows that the expected value of the area of uncertainty given at least one detection is essentially equal to the area of the detection region $\Omega_{T}$ in these regions of $S$ (recall that area $\left(\Omega_{T}\right)=A_{\Omega}=14$, for this example). Likewise, the area of uncertainty should be minimum in the region of $S$ with densest 


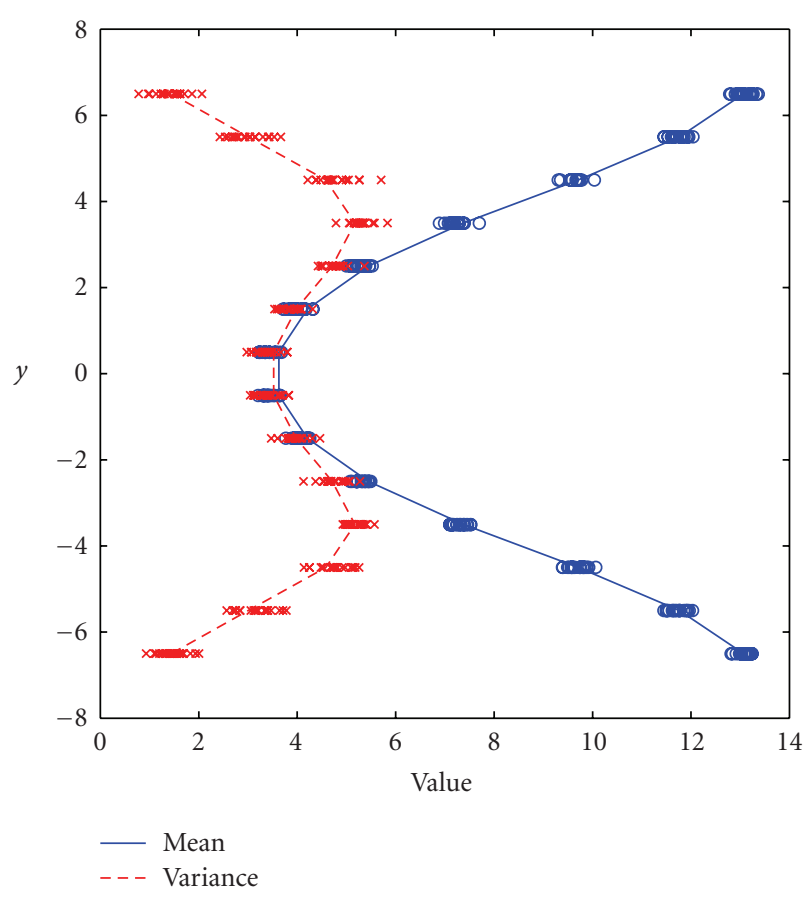

FIGURE 8: Expected value and variance of area of intersection given at least one detection, as functions of the $y$ location of the midpoint of the detection region $\Omega_{T}$, for the barrier example.

sensor coverage, which, for this example, is the line $y=0$. Indeed, the expected area of uncertainty given at least one detection for this example reaches its minimum value of 3.5552 at $y=0$.

On the other hand, the behavior of the variance of the area of uncertainty for this example, as displayed by the dashed line in Figure 8, is not so clearly anticipated. In the tails of the barrier, where few, if any, detections are expected, the variance of the area of uncertainty given at least one detection tends to zero as the target moves away from the barrier. This result is expected, since given exactly one detection, the variance is precisely zero. That the variance should increase as the target enters the barrier is also reasonable, as the uncertainty in the area of intersection $A_{\text {int }}(k)$ necessarily increases (from zero) once more than one sensor contributes to the region of intersection $\Omega_{\text {int }}(k)$, that is, for $k>1$. However, as the target approaches the center of the barrier, where the sensor density is greatest, the variance of the area of uncertainty decreases, and reaches its minimum value of 3.4601 at $y=0$. Evidently, for this example, there is a value of sensor density that, when exceeded, yields a decrease in the variance of the area of uncertainty, and otherwise leads to an increase in this variance.

\subsection{Arbitrary sensor field}

As a next example, consider sensors distributed randomly according to an arbitrary distribution function, and in particular, one for which the distributions of the $x$ and $y$ sensor locations are dependent. In this case, given the assumptions presented at the end of Section 2.1, that is, for a long, narrow detection region $\Omega_{T}$, and for a sensor location density function $f_{X Y}$ that does not vary much in the $x$ dimension (the narrow dimension of $\Omega_{T}$ ), it is reasonable to assume that the sensor $x$ and $y$ locations are locally independent in $\Omega_{T}$, so that

$$
f_{X Y \mid \Omega_{T}}(x, y) \approx f_{X \mid \Omega_{T}}(x) f_{Y \mid \Omega_{T}}(y),
$$

with the conditional density function $f_{X \mid \Omega_{T}}$ given by (18) and $f_{Y \mid \Omega_{T}}$ given by

$$
f_{Y \mid \Omega_{T}}(y)=\frac{f_{X Y}\left(X=x_{\Omega}+L_{x} / 2, y\right)}{\int_{y_{\Omega}}^{y_{\Omega}+L_{y}} f_{X Y}\left(X=x_{\Omega}+L_{x} / 2, \psi\right) d \psi},
$$

for $y_{\Omega} \leq y \leq y_{\Omega}+L_{y}$, and $f_{Y \mid \Omega_{T}}(y)=0$ otherwise. For convenience in this example, we use the fact that an arbitrary density function can be approximated to an arbitrary level of accuracy by a mixture density function (a weighted sum of density functions) with a sufficient number of terms. In particular, consider the $\mathcal{K}$ component, heterogeneous, bivariate normal mixture density function given by

$$
f_{X Y}(x, y)=\frac{1}{\mathcal{K}} \sum_{1 \leq \kappa \leq \mathcal{K}} \frac{1}{\eta_{\kappa}} \phi\left(\frac{x-\nu_{\kappa}}{\eta_{\kappa}}\right) \frac{1}{\sigma_{\kappa}} \phi\left(\frac{y-\mu_{\kappa}}{\sigma_{\kappa}}\right),
$$

with component means $v_{\kappa}$ and $\mu_{\kappa}$ in the $x$ and $y$ dimensions, respectively, with corresponding standard deviations $\eta_{\kappa}$ and $\sigma_{\kappa}$, for $\kappa=1, \ldots, \mathcal{K}$. Clearly, the $x$ and $y$ components of this density function are dependent. Given this mixture approximation to the density function $f$, and given the assumptions on the detection region $\Omega_{T}$ stated above, the conditional density function $f_{Y \mid \Omega_{T}}$, as given by (49), becomes

$$
f_{Y \mid \Omega_{T}}(y)=\frac{1}{c} \sum_{1 \leq \kappa \leq \mathcal{K}} \frac{1}{\eta_{\kappa}} \phi\left(\frac{x_{\Omega}+L_{x} / 2-v_{\kappa}}{\eta_{\kappa}}\right) \frac{1}{\sigma_{\kappa}} \phi\left(\frac{y-\mu_{\kappa}}{\sigma_{\kappa}}\right),
$$

with normalization constant $c$ given by

$$
\begin{aligned}
c= & \sum_{1 \leq \kappa \leq \mathcal{K}} \frac{1}{\eta_{\kappa}} \phi\left(\frac{x_{\Omega}+L_{x} / 2-v_{\kappa}}{\eta_{\kappa}}\right) \\
& \times\left[\Phi\left(\frac{y_{\Omega}+L_{y}-\mu_{\kappa}}{\sigma_{\kappa}}\right)-\Phi\left(\frac{y_{\Omega}-\mu_{\kappa}}{\sigma_{\kappa}}\right)\right] .
\end{aligned}
$$

The conditional distribution function $F_{Y \mid \Omega_{T}}(y)$ for $y_{\Omega} \leq y \leq$ $y_{\Omega}+L_{y}$ is obtained by integrating (51) from $y_{\Omega}$ to $y$ yielding

$$
\begin{aligned}
F_{Y \mid \Omega_{T}}(y)= & \frac{1}{c} \sum_{1 \leq \kappa \leq \mathcal{K}} \frac{1}{\eta_{\kappa}} \phi\left(\frac{x_{\Omega}+L_{x} / 2-v_{\kappa}}{\eta_{\kappa}}\right) \\
& \times\left[\Phi\left(\frac{y-\mu_{\kappa}}{\sigma_{\kappa}}\right)-\Phi\left(\frac{y_{\Omega}-\mu_{\kappa}}{\sigma_{\kappa}}\right)\right] .
\end{aligned}
$$

Substituting (53), and the conditional distribution function $F_{X \mid \Omega_{T}}(x)=\left(x-x_{\Omega}\right) / L_{x}$

for $x$ restricted to $\Omega_{T}$, into the results of Sections 2.1, 2.2, and 3 , gives expressions for the expected value and variance of the area of uncertainty $A_{\text {int }}$ for an arbitrary, but known, sensor location distribution function. 
Figure 9 shows contours of an arbitrary sensor location density function, generated using the mixture density function (50) with $\mathcal{K}=5$ components, each with equal $x$ and $y$ standard deviations $\eta_{\kappa}=\sigma_{\kappa}=2$ for all $\kappa$, and with $x$ and $y$ mean locations chosen randomly and independently from the uniform $(-10,10)$ distribution. Also shown in this figure is a sample of 50 sensors. To examine the behavior of the area of uncertainty for the constant velocity target of the previous examples for this particular sensor location density function, we evaluate the expected area of uncertainty, $E\left(A_{\text {int }} \mid 1\right)$, and the variance of this area, $\operatorname{var}\left(A_{\text {int }} \mid 1\right)$, given at least one detection, for the midpoint of the target track at $x=-9,-8, \ldots, 8,9$ and $y=-6.5,-5.5, \ldots, 5.5,6.5$. The points in this rectangular grid are chosen so that the union of the detection regions $\Omega_{T}$ centered at each point of the grid equals the search space $S$. Figures 10(a) and 11(a) show the expected value and variance, respectively, of the area of uncertainty given at least one detection from 50 sensors distributed according to the density function shown in Figure 9. These quantities are calculated at each point of the grid using the conditional sensor location distribution functions given above, and the expressions for $E\left(A_{\text {int }} \mid 1\right)$ and $\operatorname{var}\left(A_{\text {int }} \mid 1\right)$ derived in Sections 2 and 3. These theoretical results are verified experimentally by estimating $E\left(A_{\text {int }} \mid 1\right)$ and $\operatorname{var}\left(A_{\text {int }} \mid 1\right)$ using the same approach used in the previous two examples. In particular, we estimate the sample statistics $\bar{A}_{\text {int }}$ and $\bar{V}_{\text {int }}$ for $m=100050$-sensor draws and for the detection region $\Omega_{T}$ centered at each grid point. For each of the 19.14 $=266$ locations of the detection region $\Omega_{T}$ in the search region $S$, the values of $\bar{A}_{\text {int }}$ and $\bar{V}_{\text {int }}$ are plotted in Figures 10(b) and 11(b), respectively. For reference, each of the plots in Figures 10 and 11 show the same sample of 50 sensors shown in Figure 9. Also, each of these plots shows the target detection region $\Omega_{T}$ centered at the grid point with the smallest area of uncertainty, that is, the smallest value of $E\left(A_{\text {int }}\right)$; the dashed lines indicate the boundary of $\Omega_{T}$, and the arrow represents the target track over the search interval $T$.

As for the previous two examples, the estimates $\bar{A}_{\text {int }}$ and $\bar{V}_{\text {int }}$ show good agreement with the corresponding theoretical values of $E\left(A_{\text {int }} \mid 1\right)$ and $\operatorname{var}\left(A_{\text {int }} \mid 1\right)$. Also, the general trends in the expected value and variance of the area of uncertainty for this arbitrary sensor location distribution are similar to those observed for the sensor barrier. In particular, the expected area of uncertainty tends to decrease monotonically as the target approaches regions of dense sensor coverage, and increase monotonically as the target leaves these regions. Also, while there is an initial increase in the variance of the area of uncertainty as the target approaches regions of dense sensor coverage, the variance then decreases when a certain level of sensor density is exceeded. Both trends have been consistently observed for other arbitrary sensor location distributions generated from the general mixture density (50), but those results are not included here.

We finally consider a case of a sensor field with $\ell>1$ required detections. Using an increased value of $\ell$ in a field design may be performed to reduce the impact of false alarms in large fields, as pointed out in [9]. We consider a sensor field of 50 sensors randomly distributed according to the

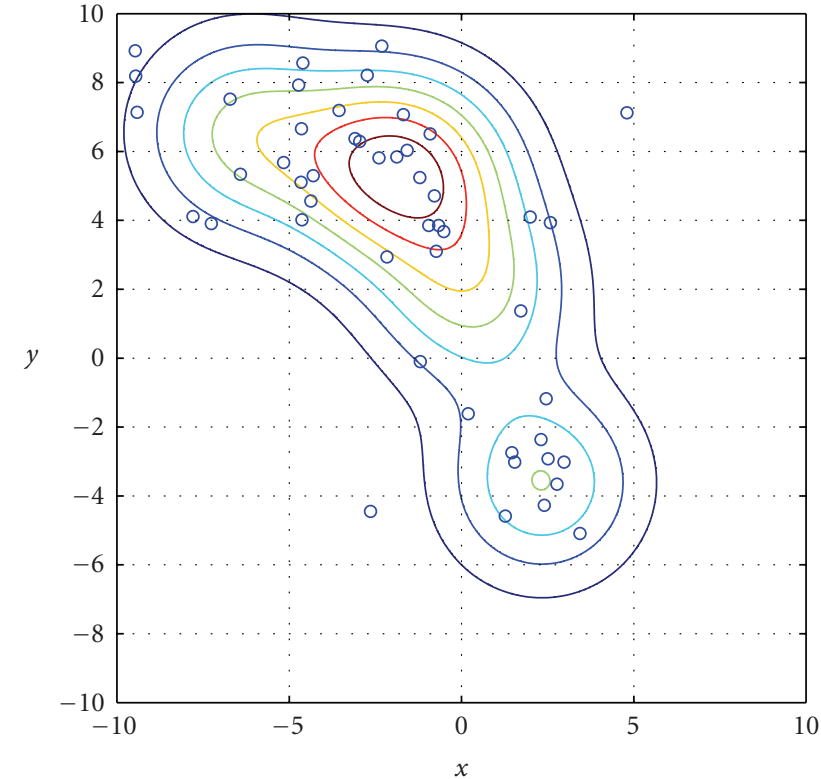

FIGURE 9: Arbitrary sensor location density function, with $N=50$ sampled sensors.

same process as the previous example. The resulting field is shown in Figure 12. As in the previous example, we examine the behavior of the area of uncertainty for the constant velocity target for this particular sensor location density function. However, in this case, we evaluate the expected value of the area of uncertainty given at least four detections, that is, $E\left(A_{\text {int }} \mid 4\right)$. The points in this rectangular grid are chosen in the same manner as for Figure 10. Figure 13 shows $E\left(A_{\text {int }} \mid 4\right)$ from 50 sensors distributed according to the density function shown in Figure 12. Note that the expected area is very small throughout much of the region, with an average that is noticeably smaller than the previous example (which had the same number of sensors, but only required a single detection). This is due to the ability of the track-before-detect kinematic requirements to ignore many detections that are not aligned with three other detections (for this $\ell=4$ case). The drawback is that it is not very likely to obtain multiple detections that provide such track information. Figure 14 shows the corresponding probability of obtaining four detections consistent with the track-before-detect criteria for this example. It is clear from the figure that the regions of highest sensor density contain both the highest probability of obtaining multiple detections and the best corresponding expected area of uncertainty. Unfortunately, as pointed out in previous work [9], these regions of high sensor density also correspond to the greatest probability of false search results. There are also many regions in Figure 13 that indicate very good localization accuracy but are very unlikely to receive the necessary detections (such as the region near $(x, y)=(-4,1)$ ).

While the preceding examples show that increased sensor density (i.e., clustering of sensors) may be beneficial to improving localization accuracy, it is important to consider this design objective in the context of other objectives. In particular, as the last example shows, better localization 


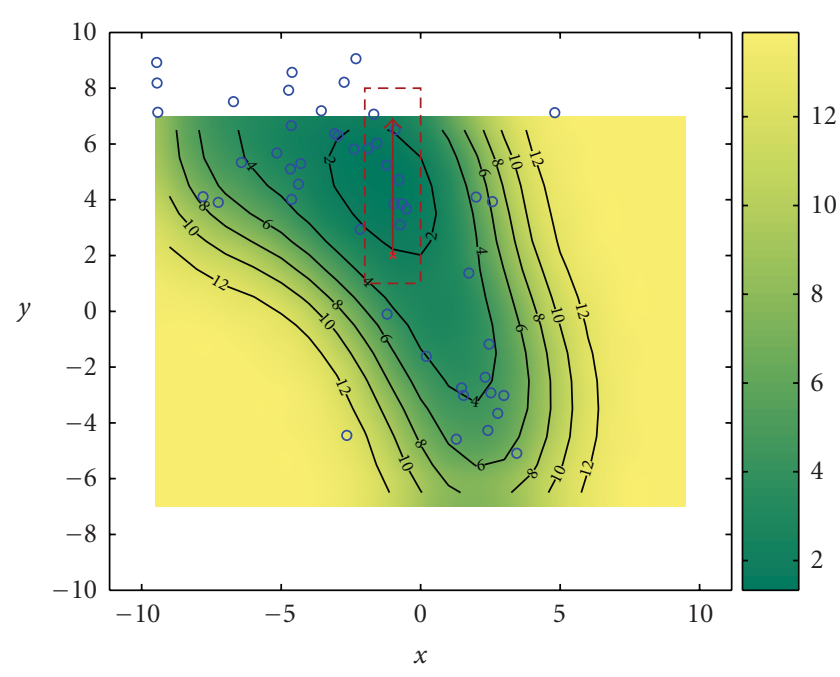

(a) Analytical result

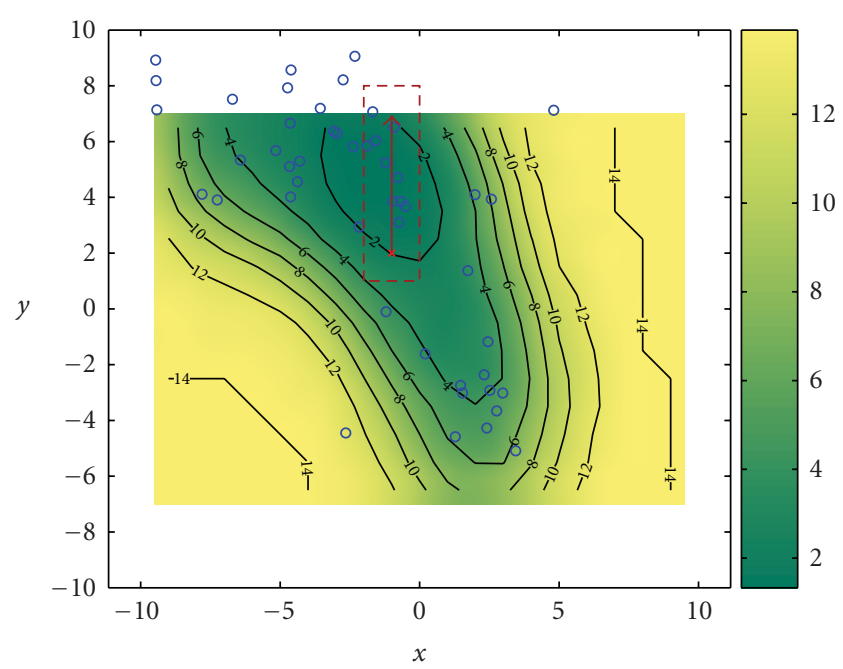

(b) Experimental result

FIgURE 10: Expected area of intersection given at least one detection.

accuracy often comes at the same locations as increased search effectiveness, but sometimes comes at locations of poor search effectiveness. Neither having good localization accuracy without detections nor having many detections without a sufficiently small area of uncertainty is useful. Even when we have both good search effectiveness and good localization, it is often due to a high density of sensors, which causes more false search reports. Thus we expect these results to be used in careful tradeoff analyses (as in [13]) to determine the best tradeoff under design constraints within each specific deployment scenario.

\section{CONCLUSIONS}

In this paper, expressions were derived for the expected value and variance of the area of uncertainty achieved by employing a track-before-detect search strategy for localizing a target moving across a distributed sensor network. The analyt-

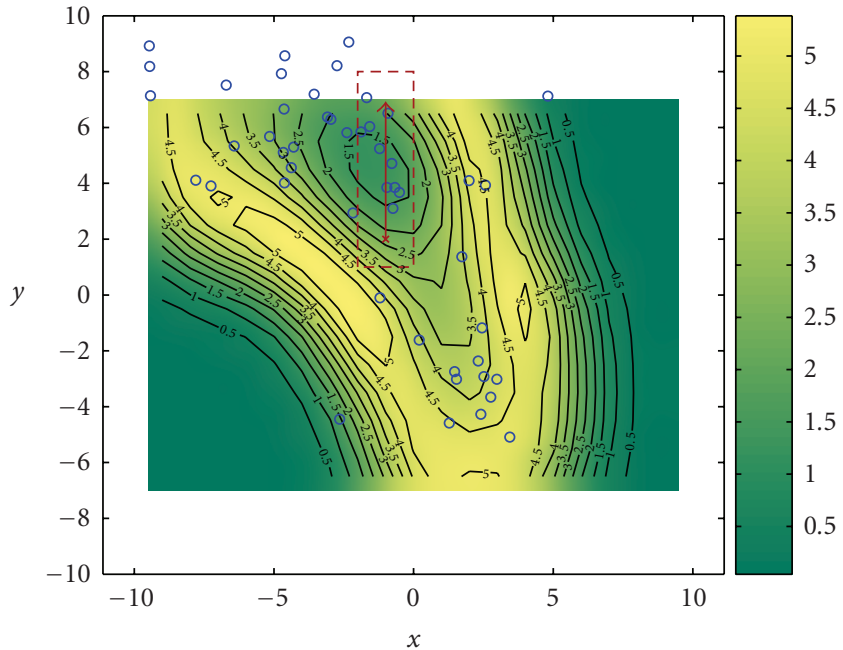

(a) Analytical result

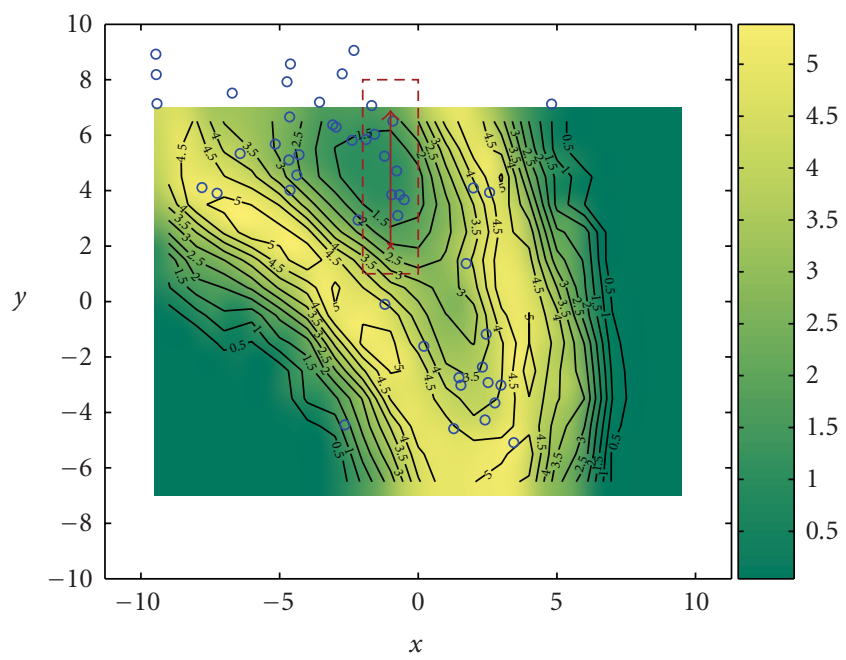

(b) Experimental result

FIGURE 11: Variance of area of intersection given at least one detection.

ical expressions were verified by comparison with computational experiments. Examples of uniform, barrier, and arbitrary field designs were analyzed using these expressions. By studying the analytical expressions for localization accuracy, system designers can develop a priori measures of effectiveness of the resulting sensor system in a parametric manner, thus enabling the optimal setting of critical design parameters, such as the placement of sensors within a search area. The analytical nature of these expressions further provides a mechanism for rapid assessment of the area of uncertainty for systems operating in real-time, which is beneficial in assessing the potential impact of field degradation on system performance. The use of these expressions within tradeoff analyses for distributed sensor system design is a subject of on-going research.

The present paper is concerned with track-before-detect that is limited to kinematic matching of expected target behavior to sensor detections. By considering the expected 


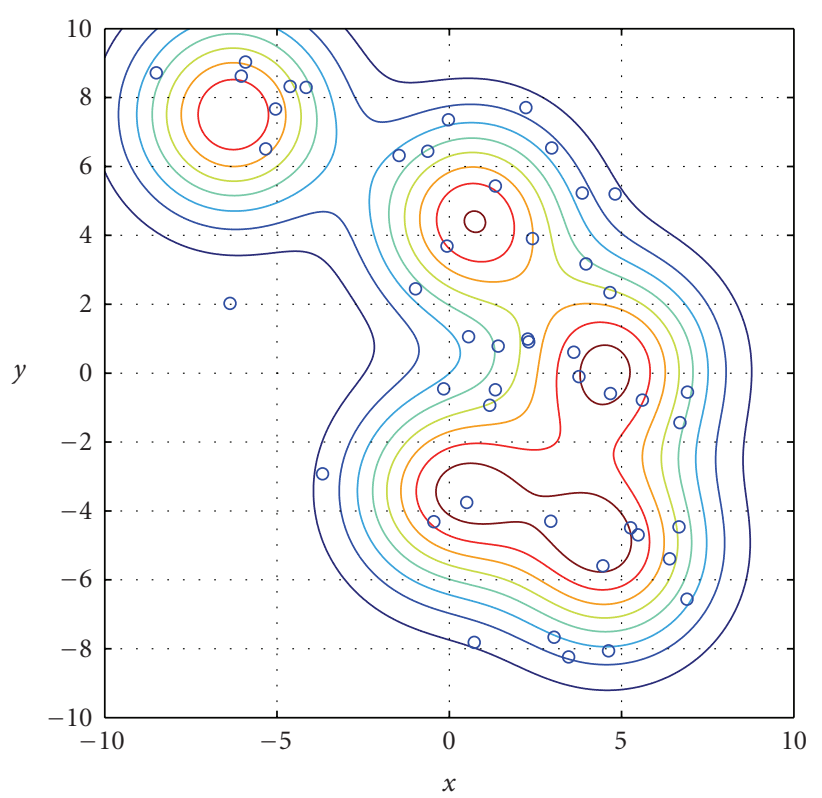

FIGURE 12: A second arbitrary sensor location density function, with $N=50$ sampled sensors.

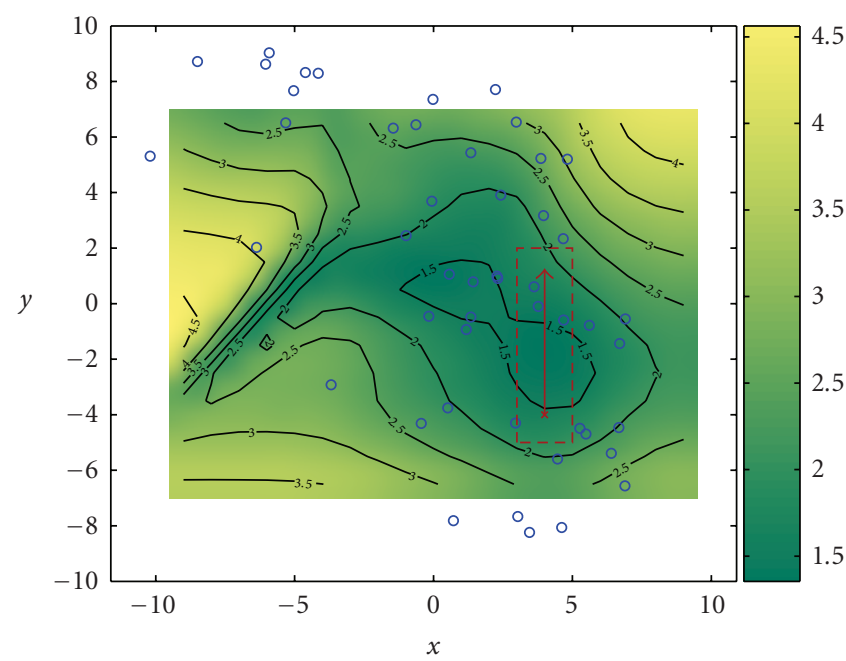

FIgURE 13: Expected area of intersection given at least four detections for the sensor density in Figure 12.

sensor detections in a probabilistic manner, this method is useful as a tool for designing sensor fields to track moving targets. The cases in this paper have been limited to single targets; the extension to multiple targets is a known benefit of track-before-detect strategies and is a subject of future interest. Other future areas of application of these results are in field design guidance that trades-off false alarm performance and expected localization accuracy, as well as the extension to heterogeneous sensor fields.

\section{APPENDIX}

Before proceeding to the proof of Lemma 2, we recall some facts about the beta distribution. The interested reader is re-

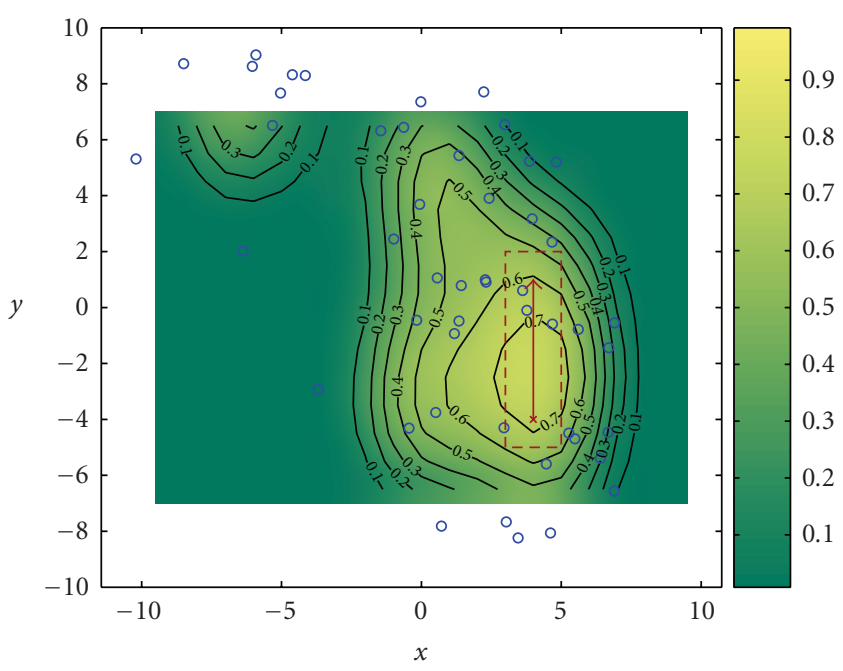

FIGURE 14: Probability of obtaining four sensor detections during a track interval for the sensor density in Figure 12.

ferred to Casella and Berger [14] for more details. The beta distribution with parameters $\lambda>0$ and $\mu>0$, denoted $\operatorname{beta}(\lambda, \mu)$, is a continuous distribution with density function $\beta(x ; \lambda, \mu)$ for $0 \leq x \leq 1$ given by

$$
\beta(x ; \lambda, \mu)=\frac{x^{\lambda-1}(1-x)^{\mu-1}}{B(\lambda, \mu)},
$$

where the constant $B(\lambda, \mu)$ can be written in terms of gamma functions, specifically $B(\lambda, \mu)=\Gamma(\lambda) \Gamma(\mu) / \Gamma(\lambda+\mu)$. Furthermore, if $X$ is a random variable distributed $\operatorname{beta}(\lambda, \mu)$, then the expected value and variance of $X$ are given by

$$
E(X)=\frac{\lambda}{\lambda+\mu}, \quad \operatorname{var}(X)=\frac{\lambda \mu}{(\lambda+\mu)^{2}(\lambda+\mu+1)},
$$

respectively. The expressions in (A.1) and (A.2) are required within the following proof. We now complete the proof of Lemma 2.

Proof of Lemma 2. Since sensor $x$ location is distributed uniform $\left(x_{\Omega}, x_{\Omega}+L_{x}\right)$ in $\Omega_{T}$, we have that

$$
F_{X \mid \Omega_{T}}(x)= \begin{cases}0, & x<x_{\Omega}, \\ \frac{\left(x-x_{\Omega}\right)}{L_{x}}, & x_{\Omega} \leq x \leq x_{\Omega}+L_{x}, \\ 1, & x>x_{\Omega}+L_{x} .\end{cases}
$$

Expressions (24) can be obtained by substituting this definition for the conditional distribution of sensor $x$ location into (15) and (22), respectively, and computing the necessary integrals. However, it is easier to first prove that the stronger statement $d_{x}(k) / L_{x}$ is distributed beta $(k-1,2)$, and then use the expressions in (A.2) for the mean and variance of a beta distributed random variable to show (24).

To show that $d_{x}(k) / L_{x}$ is distributed beta $(k-1,2)$, we start with the distribution function for the range $d_{x}(k)$, 
which we denote $G_{k}$, as given by Stuart and Ord [11, page 494]:

$$
\begin{gathered}
G_{k}(\rho)=k \int_{x_{\Omega}}^{x_{\Omega}+L_{x}}\left[F_{X \mid \Omega_{T}}(x+\rho)-F_{X \mid \Omega_{T}}(x)\right]^{k-1} \\
\times d F_{X \mid \Omega_{T}}(x), \quad 0 \leq \rho \leq L_{x} .
\end{gathered}
$$

Note that the support of the distribution function $G_{k}$ is the closed interval $\left[0, L_{x}\right]$; the endpoints of this interval are the extreme values of the range $d_{x}(k)$. From (A.3), we have, for sensor $x$ location distributed uniform $\left(x_{\Omega}, x_{\Omega}+L_{x}\right)$,

$$
d F_{X \mid \Omega_{T}}(x)= \begin{cases}\frac{d x}{L_{x}}, & x_{\Omega} \leq x \leq x_{\Omega}+L_{x}, \\ 0, & \text { otherwise }\end{cases}
$$

which creates a change of integration variable in (A.4) leading to

$$
G_{k}(\rho)=\frac{k}{L_{x}} \int_{x_{\Omega}}^{x_{\Omega}+L_{x}}\left[F_{X \mid \Omega_{T}}(x+\rho)-F_{X \mid \Omega_{T}}(x)\right]^{k-1} d x .
$$

Hence, using the partitioning from (A.3), we arrive at

$$
\begin{aligned}
G_{k}(\rho)= & \frac{k}{L_{x}} \int_{x_{\Omega}}^{x_{\Omega}+L_{x}-\rho}\left[F_{X \mid \Omega_{T}}(x+\rho)-F_{X \mid \Omega_{T}}(x)\right]^{k-1} d x \\
& +\frac{k}{L_{x}} \int_{x_{\Omega}+L_{x}-\rho}^{x_{\Omega}+L_{x}}\left[F_{X \mid \Omega_{T}}(x+\rho)-F_{X \mid \Omega_{T}}(x)\right]^{k-1} d x \\
= & \frac{k}{L_{x}} \int_{x_{\Omega}}^{x_{\Omega}+L_{x}-\rho}\left(\frac{\rho}{L_{x}}\right)^{k-1} d x \\
& +\frac{k}{L_{x}} \int_{x_{\Omega}+L_{x}-\rho}^{x_{\Omega}+L_{x}}\left(1-\frac{x-x_{\Omega}}{L_{x}}\right)^{k-1} d x \\
= & \frac{k \rho^{k-1}\left(L_{x}-\rho\right)}{L_{x}^{k}}+\frac{\rho^{k}}{L_{x}^{k}} .
\end{aligned}
$$

Then the density function of $d_{x}(k)$, denoted $g_{k}$, is given by

$$
g_{k}(\rho)=G_{k}^{\prime}(\rho)=\frac{k(k-1) \rho^{k-2}\left(L_{x}-\rho\right)}{L_{x}^{k}}
$$

for $0 \leq \rho \leq L_{x}$. Consider the density function for a $\operatorname{beta}(k-$ $1,2)$ random variable $X$; from (A.1),

$$
\beta(x ; k-1,2)=\frac{x^{k-2}(1-x)}{B(k-1,2)} .
$$

Recall that, for any positive integer $n>0$, the gamma function satisfies $\Gamma(n)=(n-1)$ !. Thus

$$
B(k-1,2)=\frac{\Gamma(k-1) \Gamma(2)}{\Gamma(k+1)}=\frac{(k-2) ! 1 !}{k !}=\frac{1}{k(k-1)} .
$$

It follows that

$$
\beta(x ; k-1,2)=k(k-1) x^{k-2}(1-x) .
$$

Comparing (A.8) and (A.11), it is clear that for $L_{x}=1$, the range $\rho=d_{x}(k)$ is distributed beta $(k-1,2)$. Suppose $L_{x} \neq 1$ and let $\xi=\rho / L_{x}=\tau(\rho)$. Then, since $\tau$ is a linear (monotonic and continuously differentiable) function of $\rho$, we have $\tau^{-1}(\xi)=\rho=L_{x} \xi$, and $\left|d / d \xi \tau^{-1}(\xi)\right|=L_{x}$. Let $f_{\Xi}$ denote the density function of the random variable $\Xi$ associated with $\xi$. It follows that

$$
\begin{aligned}
f_{\Xi}(\xi) & =g_{k}\left(\tau^{-1}(\xi)\right)\left|\frac{d}{d \xi} \tau^{-1}(\xi)\right| \\
& =\frac{k(k-1)\left(L_{x} \xi\right)^{k-2}\left(L_{x}-L_{x} \xi\right)}{L_{x}^{k}} L_{x} \\
& =k(k-1) \xi^{k-1}(1-\xi),
\end{aligned}
$$

from which we deduce that $\xi=\rho / L_{x}=d_{x}(k) / L_{x}$ is distributed beta $(k-1,2)$. Thus $d_{x}(k) / L_{x}$ is distributed beta $(k-$ $1,2)$ for all values of $L_{x}$. Substituting $k-1$ for $\lambda$ and 2 for $\mu$ in (A.2), it is straightforward to show (24).

\section{ACKNOWLEDGMENT}

This work was supported by the Office of Naval Research Code 321MS.

\section{REFERENCES}

[1] D. Li, K. D. Wong, Y. H. Hu, and A. M. Sayeed, "Detection, classification, and tracking of targets," IEEE Signal Processing Magazine, vol. 19, no. 2, pp. 17-29, 2002.

[2] F. Zhao, J. Shin, and J. Reitch, "Information-driven dynamic sensor collaboration for tracking applications," IEEE Signal Processing Magazine, vol. 19, no. 2, pp. 61-72, 2002.

[3] D. Culler, D. Estrin, and M. Srivastava, "Overview of sensor networks," Computer, vol. 37, no. 8, pp. 41-49, 2004.

[4] H. Cox, "Cumulative detection probabilities for a randomly moving source in a sparse field of sensors," in Proceedings of the 23rd Asilomar Conference on Signals, Systems and Computers, vol. 1, pp. 384-389, Pacific Grove, Calif, USA, October 1989.

[5] H. M. Shertukde and Y. Bar-Shalom, "Detection and estimation for multiple targets with two omnidirectional sensors in the presence of false measurements," IEEE Transactions on Acoustics, Speech, and Signal Processing, vol. 38, no. 5, pp. 749763, 1990.

[6] T. A. Wettergren, R. L. Streit, and J. R. Short, “Tracking with distributed sets of proximity sensors using geometric invariants," IEEE Transactions on Aerospace and Electronic Systems, vol. 40, no. 4, pp. 1366-1374, 2004.

[7] J.-P. Le Cadre and G. Souris, "Searching tracks," IEEE Transactions on Aerospace and Electronic Systems, vol. 36, no. 4, pp. 1149-1166, 2000.

[8] S. Ferrari, "Track coverage in sensor networks," in Proceedings of the American Control Conference, vol. 2006, pp. 7 pages, Minneapolis, Minn, USA, June 2006.

[9] T. A. Wettergren, "Performance of search via track-beforedetect for distributed sensor networks," to appear in IEEE Transactions on Aerospace and Electronic Systems.

[10] I. Moskowitz and J. Simmen, "Asymptotic results in search theory," Naval Research Logistics, vol. 36, no. 5, pp. 577-596, 1989.

[11] A. Stuart and J. K. Ord, Kendall's Advanced Theory of Statistics, Volume 1: Distribution Theory, Edward Arnold, London, UK, 6th edition, 1994. 
[12] W. Feller, An Introduction to Probability Theory and Its Applications, vol. 1, John Wiley \& Sons, New York, NY, USA, 1968.

[13] T. A. Wettergren, R. Costa, J. G. Baylog, and S. P. Grage, "Assessing performance tradeoffs in undersea distributed sensor networks," in Proceedings of the IEEE Oceans Conference (OCEANS '06), pp. 1-6, Boston, Mass, USA, September 2006.

[14] G. Casella and R. L. Berger, Statistical Inference, Duxbury Press, Belmont, Calif, USA, 1990. 